\title{
Pronominale Referenz im Jiddischen und Deutschen im 21. Jahrhundert
}

Steffen Krogh \& Kathrine Thisted Petersen

Aarhus Universitet

\begin{abstract}
Osteuropäisches Jiddisch und Deutsch gehören von Hause aus zu denjenigen germanischen Sprachen, die bei pronominaler Referenz strenge Genuskongruenz wahren: Als Faustregel galt bisher, dass auf jedwedes Bezugswort stets mittels der Formen er/er, zi/sie und es/es verwiesen wurde, gleichgültig, ob das Bezugswort einen Menschen, ein Tier oder sonstiges Lebewesen, einen Gegenstand oder ein Abstraktum bezeichnete. ${ }^{1}$ Die vorliegende Arbeit ist der Frage gewidmet, wie sich die beiden Sprachen in jüngerer bzw. jüngster Zeit von dieser gemeinsamen Grundlage entfernt haben. Als spezieller Vertreter des Jiddischen dient das vorrangig in den USA beheimatete charedische (ultraorthodoxe) Satmarer Jiddisch, das im 21. Jahrhundert die sprecherstärkste Varietät des Jiddischen ausmacht. Anhand ausgewählter Belege aus den letzten 100 Jahren für das Jiddische bzw. dem letzten Jahrzehnt für das Deutsche werden sowohl Gemeinsamkeiten als auch Unterschiede hinsichtlich der Art und des Umfangs dieser Neuerung in den zwei Sprachen herausgearbeitet.
\end{abstract}

\section{Der Stand der Dinge im ausgehenden 20. Jahrhundert}

Unter pronominaler Referenz ist die Verbindung zu verstehen, die der Sprecher beim Rückverweis, Vorwärtsverweis sowie in deiktischer Verwendung (mit oder ohne Begleitgeste) zwischen einem Pronomen und dem Bezugswort herstellt, das vom fraglichen Pronomen ersetzt wird. Bei deiktischer Verwendung braucht das Bezugswort nicht explizit geäußert worden zu sein; es kann auch lediglich mitgedacht sein. In

1 Die Transkription des Ostjiddischen folgt der Umschrift des YIVO Institute for Jewish Research (Weinreich 1999: 26).

Ken Ramshøj Christensen, Henrik Jørgensen \& Johanna L. Wood (eds.). 2019.

The Sign of the V-Papers in Honour of Sten Vikner. Dept. of English, School of Communication \& Culture, Aarhus University, pp. 405-418, doi:10.7146/aul.348.106. (C) The author(s). 
allen germanischen Einzelsprachen galt ursprünglich das aus dem Urgermanischen ererbte Prinzip, wonach ein Personalpronomen auf jedwedes maskuline, feminine und neutrale Bezugswort verwies. Die Referenz erfolgte unabhängig davon, ob mit dem fraglichen Bezugswort ein Mensch, ein Tier oder sonstiges Lebewesen, ein Gegenstand oder ein Abstraktum bezeichnet wurde.

Bisher gehören das osteuropäische Jiddisch (hinfort: Jiddisch) und das Deutsche $\mathrm{zu}$ den vergleichsweise wenigen germanischen Sprachen, die das ererbte Verweissystem bis in die Gegenwart weitergeführt haben. So referieren jidd. er, $z i$ und dt. er, sie nicht nur auf Menschen wie im Falle von jidd. man ,Mann', froy, Frau', dt. Mann, Frau, sondern auch auf Tiere wie jidd. vorem ,Wurm', shlang ,Schlange', dt. Wurm, Schlange, Sachen wie jidd. tish ,Tisch', brust ,Brust', dt. Tisch, Brust und Abstrakta wie jidd. gloybn ,Glaube', freyd ,Freude', dt. Glaube, Freude. Mithin lässt sich mit der Frage jidd. vu iz er/zi?, dt. Wo ist er/sie? der Aufenthaltsort nicht nur von Menschen, sondern auch von anderen Bezugsgrößen erfragen, die in der betreffenden Sprechsituation erscheinen oder erschienen sind. Ausnahmen von dieser eisernen Regel können insbesondere im Bereich der Constructio ad sensum auftreten, und zwar dann, wenn Genus und Sexus auseinandergehen. So besteht bei jidd. meydl ,Mädchen', dt. Mädchen neben der stets grammatikalisch korrekten Genuskongruenz, die hergestellt wird, indem mit jidd. es, dt. es verwiesen wird, auch die Möglichkeit, mit jidd. $z i$ bzw. dt. sie auf das Bezugswort zu verweisen. Constructio ad sensum kann in diesem Fall auf eine lange Vorgeschichte zurückblicken (dazu mit reichhaltigem historischem Belegmaterial Behaghel 1928: 3839).

Es folgt nun eine Reihe authentischer Belege aus beiden Sprachen, mit denen die Festigkeit des ererbten Verweissystems exemplarisch vorgeführt werden soll (Bezugswort und Pronomen in Fettdruck):

\section{A. Verweis auf Tiere:}

(1) Jiddisch

azoy, zog ikh, ihr meynt dos ernst? vifiel, lemoshl, kost ayer sus? in vifiel, zogt er, shatst ihr ihm? [...] tselakht er zikh un zogt, az er kost mehr fun dray mol azoy fiel (Sholem Aleykhem 1925: 152). ,Ach so, sage ich, meinen Sie das ernst? Wieviel kostet zum Beispiel Ihr Ross? Auf wieviel, sagt er, schätzen Sie es? Dann bricht er in Gelächter aus und sagt, dass es mehr als dreimal so teuer ist'. 


\section{(2) Jiddisch}

oft iz oysgekumen tsu shlepn a $\boldsymbol{k} \boldsymbol{u}$ un $\boldsymbol{z} \boldsymbol{i}$ tsu shlogn mit a palke (Bashevis 1980: 72).

,Oft musste man eine Kuh hinter sich herziehen und sie mit einem Stock schlagen'.

\section{(3) Deutsch}

Nur wenige Vögel überleben den Crash. Zwar flattert der ein oder andere noch fort ins Gebüsch, meist aber stirbt er dort an seinen Verletzungen (Der Spiegel, 2, 07.01. 2017: 110,2).

\section{(4) Deutsch}

Schmuckschildkröte. Eine der am häufigsten gehandelten Schildkröten überhaupt - in der EU ist sie als Schädling eingestuft worden und soll in den Mitgliedsländern bekämpft werden (Die Zeit, 03.08. 2017: 32,1).

\section{B. Verweis auf Gegenstände}

\section{(5) Jiddisch}

[...] alts breyter iz gevorn der taykh. er hot zikh oysgeshpreyt vi a shpigl (Bashevis 1980: 247).

,[...] umso breiter wurde der Fluss. Wie ein Spiegel breitete er sich aus 6

\section{(6) Jiddisch}

me vet mir ophaken di hand, di hand, vos zi hot ge'ganve't (Sholem Aleykhem 1927: 21).

,Man wird mir die Hand abhacken, die Hand, die gestohlen hat" (mit $z i$ als Resumptivum).

\section{(7) Deutsch}

Chambers durfte den Speer ansehen, ihn sogar halten. "Er war schwerer, als ich gedacht hätte, und er wirkte richtig alt." Für genauere Untersuchungen mitnehmen durfte er ihn dann aber doch nicht (Die Zeit, 28.05. 2014: 37,4). 


\section{(8) Deutsch}

Auf dem Weg zum Treffen hatte er in einer großen Buchhandelskette versucht, den Bestseller der Saison zu erwerben, die kommentierte Ausgabe von "Mein Kampf". "Die Verkäuferin sah mich an, als hätte ich nach Kinderpornografie verlangt!" Er hat sie natürlich nicht bekommen, sie war nicht vorrätig (Der Spiegel, 19, 07.05. 2016: 126,1).

\section{Verweis auf Abstrakta}

(9) Jiddisch

mit aza kuk, vos ikh vel ihm eybig nisht fargesen (Sholem Aleykhem 1925: 113).

,Mit einem Blick, wie ich ihn niemals vergessen werde' (mit ihm als Resumptivum).

\section{(10) Jiddisch}

zi iz shoyn geven a vaybl un hot nokh alts nisht gekrogn di tsayt. shpeter, az zi hot zi gekrogn, hot zi geblutikt vi, lehavdl, a beheyme tsu der skhite (Bashevis 1980: 53).

,Sie war schon eine verheiratete Frau, hatte aber noch nicht ihre Menstruation bekommen. Als sie sie später bekam, blutete sie - man möge mir den Vergleich verzeihen - wie ein Tier auf der Schlachtbank'.

\section{(11) Deutsch}

Den Japanern aber geht es gut, materiell gesehen. Ihr Wohlstand bleibt groß, nur wächst er nicht mehr (Die Zeit, 05.06. 2014: 13,1).

\section{(12) Deutsch}

Das Schöne an Geschichte, sagt Altmaier, sei, dass sie im Gegensatz zur Politik abgeschlossen ist (Die Zeit, 07.03. 2019: 4,4). 


\section{Fälle, in denen Genus und Sexus des Bezugswortes nicht übereinstimmen}

\section{(13) Jiddisch}

er hot nokh rekht nisht gehat gezen zayn kind un er hot gegart es ontsukukn (Bashevis 1980: 261).

,Er hatte sein Kind noch nicht wirklich gesehen, und er sehnte sich danach, es zu betrachten'.

\section{(14) Jiddisch}

dayn kind, zogt er, rayst zikh in eyn ander velt arayn, un du farshtehst zi nit (Sholem Aleykhem 1925: 128).

,Dein Kind, sagt er, wirft sich in eine andere Welt, und du verstehst es nicht'.

\section{(15) Deutsch}

Dann habe er das Mädchen getötet, um zu verhindern, dass es ihn anzeigt (MOZ.de Das Nachrichtenportal für Brandenburg).

\section{(16) Deutsch}

Tag und Nacht schrie das Mädchen. Das einzige Mittel, sie zur Ruhe zu bringen, war das Fläschchen (Facebook).

(Jidd. kind, dt. Mädchen sind beide Neutra. In den obigen Belegen wird teils mit, teils ohne Genuskongruenz auf sie verwiesen).

\section{Neue Wege pronominaler Referenz}

Der Forschung ist es bereits seit einigen Jahren gut bekannt, dass das charedische (ultraorthodoxe) Satmarer Jiddisch, die jiddische Varietät, die heute von den meisten Muttersprachlern des Jiddischen gesprochen wird, nicht mehr dem obengenannten ererbten Verweisprinzip gehorcht. An seine Stelle ist ein System pronominaler Referenz getreten, das auf den ersten Blick an dasjenige des modernen Englisch erinnert: Die Formen $e r$ und $z i$ referieren wie engl. he und she fast ausschließlich auf menschliche Bezugswörter, während alle anderen Substantive durch die Neutrumformen es und $d s^{2}$, parallel zu engl. it, ersetzt werden (dazu zuletzt Krogh 2015: 398-401). Die in und um New York City beheimatete

2 dos fungiert in solchen Fällen z. T. als Personalpronomen. 
Presse der charedischen Satmarer Juden bietet eine Fülle von Belegen für dieses Verweisprinzip. Man vergleiche z. B. (Bezugswort und Pronomen in Fettdruck):

(17) ven a mentsh kumt tsum heyligen khoyze, nemt er zayn neshome un vasht dos oys un reynikt [sic] dos fun yede shmits un rost (Die vokh: 19,1).

,Wenn ein Mann zum Heiligen Seher kommt, nimmt er seine Seele, wäscht sie und säubert sie von jedem Schmutz und Rost".

(18) ,ikh bin oyf aykh goyzer az als pikuekh nefesh zolt ir esn dem zup“, der rebe hot keyn breyre nisht gehat un er hot dos gegesn (Der idisher gedank: 29,1).

,Ich befehle Ihnen, gemäß dem Gebot, menschliches Leben zu retten, die Suppe zu essen. Der Rabbiner hatte keine Wahl und aß sie'.

(19) ir vet trefn nokh a farmakhtn briv inem konvert. git es iber ,perzenlikh “ far mr. lindberg (Der blat: 58,4).

,Sie werden noch einen versiegelten Brief in dem Umschlag finden. Übergeben Sie ihn Herrn Lindbergh persönlich`.

(20) nokh an interesante teve vos di vol farmogt, dos ven es tsit arayn in zikh flisigkayt, blaybt es nisht ineveynig oyf lang, un es geyt oykh nisht tsurik aroys di fisigkayt fun vu es kumt orginal (Der id, A: 31,3).

,Noch eine interessante Eigenschaft, die Wolle besitzt, [ist,] dass wenn sie Flüssigkeit in sich zieht, diese nicht lange darin bleibt, und die Flüssigkeit geht auch nicht dorthin zurück, woher sie ursprünglich kam'.

Von den obengenannten Bezugswörtern ist briv von Hause aus maskulin, während neshome, zup, vol und flisigkayt feminin sind. Heute tritt die historische Genuszuweisung dieser Substantive morphologisch nicht mehr in Erscheinung.

Auf den ersten Blick böte es sich an, die veränderte pronominale Referenz auf den umfassenden Genussynkretismus im charedischen Satmarer Jiddisch zurückzuführen. Es handelt sich dabei in erster Linie um den Verlust von Genusmarkierungen in der Flexion des bestimmten Artikels, attributiver Adjektive, gewisser Pronomina und des Zahlwortes 
eyner, einer'. In der gesprochenen Sprache ist der Synkretismus nicht nur von Genus-, sondern auch von Kasusunterschieden so weit fortgeschritten, dass der bestimmte Artikel einheitlich [də] lautet und die anderen vorher genannten Wörter ebenfalls einheitlich auf [ə] enden. Demnach wäre anzunehmen, dass wenn Genusunterschiede verschwinden, die gewöhnliche Genuskongruenz zwischen einem Pronomen und dessen Bezugswort ihren Sinn verliert, und eine neue Differenzierung zwischen menschlich und nichtmenschlich entsteht, die es natürlich erscheinen lässt, mit einer Neutrumform auf nichtmenschliche Bezugswörter zu verweisen.

Einer derartigen Ansicht von der Abfolge der Ereignisse widerspricht freilich die von Corbett (1979: 204, 216, 218) aufgestellte Allgemeingültigkeit beanspruchende Kongruenzhierarchie. Ausgehend von in vielen Sprachen gesammelter Evidenz zieht Corbett den Schluss, dass der Verlust von Genuskongruenz bei pronominaler Referenz im Regelfall dem Verlust der Genusflexion attributiver Wörter zeitlich vorausgeht. Der Grund dafür ist Corbett zufolge in dem Umstand zu suchen, dass die faktische Entfernung zwischen Kopf (controller) und Dependens (agreeing element) bei pronominaler Referenz normalerweise größer ist als in anderen Fällen, in denen Genuskongruenz realisiert wird oder werden kann. Die Auffassung, nach der im charedischen Satmarer Jiddisch der Wandel in pronominaler Referenz der Nivellierung von Genusund Kasusunterschieden bei attributiven Wörtern zeitlich vorausgegangen sein muss, scheint durch die Untersuchungen von Krogh (2012: 496-504) zur europäischen Ausgangmundart des charedischen Satmarer Jiddisch, dem sogenannten unterländischen Jiddisch (dazu grundsätzlich Weinreich 1964), eine vorläufige Bestätigung erfahren zu haben. Damit wäre auch die Annahme hinfällig, wonach Veränderung im Bereich pronominaler Referenz im charedischen Satmarer Jiddisch auf Einfluss seitens der englischen Umgebungssprache zurückzuführen ist. Dem Englischen wäre demnach nur eine einen schon im Gang befindlichen Wandel beschleunigende Einwirkung zuzubilligen.

Der germanistischen Fachwelt dürfte jedoch weniger bzw. gar nicht bekannt sein, dass sich ein Wandel wie der oben dargelegte in Ansätzen auch im heutigen Deutsch anzubahnen scheint. Dieser Sachverhalt ist der Aufmerksamkeit der bisherigen Forschung zur modernen Syntax im Allgemeinen und zum Thema pronominale Referenz im heutigen Deutsch im Besonderen anscheinend komplett entgangen (Marga Reis, brieflich; man vergleiche auch: Bærentzen 1987: 344; Askedal 1990: 214 (dort auch die vorgängige Spezialliteratur zu dt. es); Zifonun [u. a.] (Hgg.) 
1997: 544-550; Engel 2009: 455-456; Eisenberg 2013: 173; Hentschel/ Weydt 2013: 223; Wöllstein (Hg.) 2016: 1011; Helbig/Buscha 2017: 543). Uns dafür sensibilisiert hatte die besonders im gesprochenen Deutsch immer häufiger anzutreffende Erscheinung, dass das als Demonstrativund Personalpronomen bisweilen satzübergreifend auf nichtmenschliche Bezugswörter im Maskulinum oder Femininum verweisen kann. Als erste Stufe einer gegenwärtig nur auf dem Reißbrett befindlichen großangelegten Untersuchung haben wir eine Reihe von muttersprachlichen Gewährsleuten aus Nord- und Süddeutschland sowie Österreich und der Schweiz im Alter zwischen 25 und 60 Jahren befragt, die alle bestätigen, dass ein derartiger Verweis in Situationen wie der folgenden möglich ist: Sprecher A zeigt mit dem Finger auf einen Apfel (Maskulinum) und sagt zu Sprecher B: Nimm das! Ebenso wie wenn auf die Frage: Spanischer Rotwein? geantwortet wird: Das trinke ich nicht. Im ersten Fall wird deiktisch, im zweiten Fall generisch auf die Bezugsgröße verwiesen. Mit dem Terminus generisch wird der nicht auf ein Einzelexemplar, sondern auf die Gattung fokussierende Gebrauch der Anapher bezeichnet.

Skeptischer waren unsere Gewährsleute hingegen, als danach gefragt wurde, ob in solchen Situationen (ohne Topikalisierung) nicht das, sondern es als Anapher (oder Katapher) eingesetzt werden könnte. Unter Sprechern aus Österreich und der Schweiz scheint eine größere Akzeptanz in Bezug auf diese Konstruktion zu herrschen als unter Sprechern aus Deutschland. Ersterer Sprechertyp scheint auch das obige das häufiger zu verwenden bzw. zu akzeptieren als aus Deutschland stammende Sprecher.

Da Art und Häufigkeit des hier interessierenden Verweisprinzips für das Deutsche wie erwähnt noch völlig unerforscht sind, befinden sich unsere Gedanken darüber gegenwärtig auf der Stufe von Vorüberlegungen, und es mögen die hier vorgelegten Belege und geäußerten Vermutungen einstweilen Genüge tun. Es sollen nun die folgenden von uns festgehaltenen zugegebenermaßen wenig zahlreichen Hör- und Schriftbelege präsentiert werden (Bezugswort und Pronomen in Fettdruck): 


\section{A. Belege für phorisches das}

(21) Den Becher [mit dem Konterfei Karl-Theodor zu Guttenbergs] habe ich gerade nachträglich zur Disputation von einer Freundin geschenkt bekommen. Direkt nach der Disputation wollte sie mir das nicht schenken, aus Angst, ich würde es nicht lustig finden (Hörbeleg vom 19.06. 2014).

(22) $[\ldots]$ nein, aber $L[\ldots]$ hat sich ausführlich damit beschäftigt (und eine Rezension verfasst/bzw. soll verfassen, die in dr [sic] ZDL erscheinen soll). Vielleicht fragst du sie, ob sie dir das schickt?

(Auszug aus einer E-Mail vom 30.08. 2017).

\section{B. Belege für phorisches es}

(23) Der erste Käse. In Polen fanden sich die ältesten Spuren - aber wer hat's erfunden? (Die Zeit, 13.12. 2012: 39,5). (Überschrift; wer hat's erfunden ist womöglich eine Anspielung auf den bekannten gleichlautenden Werbespot des Schweizer Bonbonherstellers Ricola).

(24) Wenn es eine glatte Schale hat, die Orange, dann ist sie sehr viel saftiger (kataphorisch, audiovisueller Beleg auf Markt (ndr.de), 03.02. 2014, 20:15 Uhr).

(25) Der Zentralfriedhof - kommt es gleich? (Hörbeleg vom 20.03. 2019).

(26) Post ist leider keine angekommen - wann hast du es denn abgeschickt? (mit Bezug auf ein als implizites Bezugswort anzusetzendes Postsendung, Chatbeleg vom 04.04. 2019).

Als besonders aufschlussreich betrachten wir den folgenden Beleg, in dem beide Verweistypen anscheinend gleichberechtigt nebeneinanderstehen:

(27) Knapp gesagt: Für Mord braucht es einen triftigen Grund und die Gelegenheit, das zu verwirklichen. Um zum Mörder zu werden, braucht es ein Selbstkonzept, das einem die Tötung erlaubt, sie rechtfertigt und womöglich schönredet (Die Zeit, 14.02. 2019: 12,2). 
Beim Rückbezug von das und sie auf Mord bzw. Tötung handelt es sich um generische Referenz. Bezeichnet werden damit nicht Einzeltaten, sondern die Verübung von Mord und Totschlag im Allgemeinen. Die meisten muttersprachlichen Gewährsleute, denen der Beleg vorgelegt wurde, betrachten im ersten Fall (das $\rightarrow$ Mord) das und ihn als mehr oder weniger gleichberechtigte Anaphern, begegnen aber im zweiten Fall (sie $\rightarrow$ Tötung) der Ersetzung der Anapher sie durch es oder das mit Skepsis bis Ablehnung. Der Grund dafür ist nicht unmittelbar einleuchtend. Nach Ansicht eines der Befragten könnte die topologische Nähe der Anapher zum Bezugswort Tötung ein Hindernis darstellen.

Gemeinhin wird in Sätzen, in denen das so viel wie ,so etwas' / ,dergleichen' bedeutet und die Bezugsgröße als entsprechend unscharf erscheint (vgl. Bærentzen 1987: 345-346), der Gebrauch des Neutrums von Muttersprachlern des Deutschen zumeist nicht nur akzeptiert, sondern geradezu als einzige Möglichkeit angesehen; man vergleiche z. B.:

(28) Vor ein paar Jahren hatten wir die Debatte um Vergewaltigung in der Ehe, und die Union vertrat den Standpunkt, das gäbe es gar nicht (Die Zeit, 16.05. 2013: 6,5).

Diese Art pronominaler Rückbezug könnte gut den Ausgangspunkt dafür gebildet haben, dass der Gebrauch von das und es als auf maskuline und feminine Bezugswörter verweisenden Anaphern (oder Kataphern) in der deutschen Gemeinsprache überhaupt erst möglich wurde und jetzt, wie es scheint, immer stärker um sich greift. ${ }^{3}$

In den oben angeführten signifikanten Belegen für phorisches das/ es (21-26) wäre dessen Ersetzung durch so etwas / dergleichen kaum möglich. das/es scheint in allen angeführten Fällen mit dem Bezugswort deckungsgleich zu sein.

In einer Hinsicht, die in der vorliegenden Studie bisher unerwähnt geblieben ist, unterscheidet sich das hier interessierende alternative Verweisprinzip im charedischen Satmarer Jiddisch frappant von seinem Gegenstück im Deutschen: beim Verweis auf ein Bezugswort im Plural. Während das klassische Ostjiddisch und das Deutsche hier nur eine Pluralform des phorischen Pronomens erlauben, folgt das charedische

3 Man vergleiche etwa folgendes Statement der Gewährsperson E.L., 53jährige Lehrerin aus dem südlichen Niedersachsen, vom 10.08. 2019: „Ja, diese Erscheinung gibt es - und ich selber verwende sie oft genug. Bei meinen Schülern allerdings finde ich sie in einer Form gehäuft vor, dass es weh tut". 
Satmarer Jiddisch einem Usus parallel zu demjenigen im Singular. Wenn das Bezugswort menschlich ist, steht das phorische Pronomen auch im Plural; wenn es dagegen eine nichtmenschliche Bezugsgröße bezeichnet, besteht zumindest die Möglichkeit, mit es/dos als Anapher (oder Katapher) darauf zu verweisen. Man vergleiche folgende Belege:

\section{Jiddisch}

(29) tsulib dem hoben zikh di mashinen asakh shneler tsibrokhen hot men dos gebrengt tsu farekhten (Die vokh: 14,5).

,Deshalb gingen die Autos viel schneller kaputt. Dann gab man sie in Reparatur'.

(30) ikh hof az m'vet mekabl zayn di verter vos m'hot do geshmuest, m'vet es mekabl zayn tsu farbesern di maysim (Der idisher gedank: 19,2). ,Ich hoffe, dass man die Worte beherzigen wird, die man hier gesprochen hat. Man wird sie beherzigen, um die Taten zu verbessern ".

(31) geyendig aroys fun ofis hot er zikh dermant az di oybershte shuflodn hot er nisht unterzukht. er efnt dos oyf (Der blat: 56,2).

,Als er das Büro verließ, erinnerte er sich daran, dass er die obersten Schubladen nicht untersucht hatte. Er öffnet sie'.

(32) ven der mentsh vert elter, farlirn di disks a teyl funem vaser vos ligt gevenlikh derin. dos makht es mer boygzam un mer oysgeshtelt az es zol zikh tseraysn (Der id, A: 36,4).

,Wenn der Mensch älter wird, verlieren die Bandscheiben einen Teil der Flüssigkeit, die sich gewöhnlich darin befindet. Das macht sie biegsamer und reißanfälliger‘.

(33) zey hobn etlikhe mol gezen fun dervaytns gantse stades ,bizons', di amerikaner rufn es bufel oks (Berman 2001: 316).

,Mehrmals sahen sie von Weitem ganze Herden ,Bisons'; die Amerikaner nennen sie Büffel'.

Wie (33) zeigt, kommen hier auch Tiere als Bezugswörter in Frage. Mit dieser Art pronominaler Referenz steht das charedische Satmarer Jiddisch im Rahmen des Ostjiddischen nach dem Stand unserer bisherigen Ermittlungen allein. Die Tatsache, dass dem unterländischen Jiddisch, der europäischen Vorstufe des charedischen Satmarer Jiddisch, 
pronominale Referenz mittels der neutralen Pronominalformen es und dos bei pluralischen Bezugswörtern komplett abgeht, lässt darauf schließen, dass diese Art pronominaler Referenz im charedischen Satmarer Jiddisch erst in den Jahren bzw. Jahrzehnten nach der Gründung der Siedlung der charedischen Satmarer Juden in New York im Jahre 1947 entstand. Im Englischen, das in Sachen pronominaler Referenz bei singularischen Bezugswörtern typologisch in jeder Hinsicht mit dem charedischen Satmarer Jiddisch übereinstimmt, fehlt von einer Übertragung besagten Prinzips auf pluralische Verweissituationen jede Spur.

\section{Zusammenfassung und Ausblick}

Beim Thema pronominale Referenz bietet sich ein Vergleich zwischen Jiddisch und Deutsch nicht zuletzt deshalb an, weil es sich bei Jiddisch und Deutsch um zwei sehr eng verwandte Sprachen handelt. In der vorliegenden Studie wurde anhand eines Belegmaterials gezeigt, wie pronominale Referenz speziell auf nichtmenschliche Bezugsgrößen im Maskulinum und Femininum teils nach der aus dem Urgermanischen ererbten strengen Genuskongruenz, teils mittels neutraler Pronominalformen in den zwei Sprachen erfolgt bzw. erfolgen kann. Trotz aller Gemeinsamkeiten in Bezug auf Entwicklungstendenzen in beiden Sprachen in den letzten Jahrzehnten bleibt festzuhalten, dass was sich im charedischen Satmarer Jiddisch, auf das oben ein spezielles Augenmerk gerichtet wurde, als Regel herausstellt, sich im Deutschen lediglich als eine Tendenz beschreiben lässt, deren Domäne derzeit eher in der gesprochenen als in der geschriebenen Sprache liegt. Ob diese Tendenz zukunftsweisend ist, bleibt ungewiss. Eine großangelegte Untersuchung der Erscheinung im gesprochenen wie geschriebenen Deutsch steht noch aus. 


\section{Literatur}

\section{A. Quellentexte}

\section{a. Belletristik}

\section{Jiddisch:}

Bashevis, Yitskhok. 1980. Der knekht. Tel-Oviv: Y.L. Perets farlag.

Berman, Yoyel. 2001. Arum di velt in akhtsig teg. A shpanende ertseylung ful mit shturemdige momentn, vos hot zikh opgeshpilt far mer vi 130 yor tsurik, ven an englisher dzentlman hot zikh farmostn arumtsuforn di gantse velt in bloyz akhtsig teg, an umderherte ershaynung in yene tsayt. Bruklin, NY: Hoytsoes kheyn.

Sholem Aleykhem. 1925. Ale verk fun Sholem-Aleykhem. Bd. 1. Gants Tevye der milkhiger. Nyu York: Sholem-Aleykhem folksfond oysgabe.

Sholem Aleykhem. 1927. Ale verk fun Sholem-Aleykhem. Bd. 7. Mayses far idishe kinder. Ershtes bukh. Nyu-York: Sholem-Aleykhem folksfond oysgabe.

\section{b. Zeitschriften}

\section{Jiddisch:}

Der idisher gedank, 2, 18.10. 1999.

\section{Deutsch:}

Der Spiegel, 19, 07.05. 2016.

Der Spiegel, 2, 07.01. 2017.

\section{c. Zeitungen}

\section{Jiddisch:}

Der blat. Tsaytshrift fun alveltlikhn ortodoksishn idntum. 15.06. 2001.

Der id. Organ fun umophengigen ortodoksishen identum in Amerike. 21.03. 2008. Teil A-B. Beilage Der shiker'er id.

Die vokh. 30.07. 1998.

\section{Deutsch:}

Die Zeit. 13.12. 2012.

Die Zeit. 28.05. 2014.

Die Zeit. 05.06. 2014.

Die Zeit. 03.08. 2017.

Die Zeit. 14.02. 2019.

Die Zeit, 07.03. 2019.

Die bibliografischen Einzelheiten von Hör- und audiovisuellen Belegen werden an Ort und Stelle mitgeteilt.

\section{d. Internetquellen}

\section{Deutsch:}

Facebook.

URL: https://www.facebook.com/ksta.fb/posts/10156179208467951/ (Stand: 14.09. 2019). 
MOZ.de Das Nachrichtenportal für Brandenburg.

URL: https://www.moz.de/nachrichten/brandenburg/artikel-ansicht/ $\mathrm{dg} / 0 / 1 / 1745164 /$ (Stand: 14.09. 2019).

\section{B. Sekundärliteratur}

Askedal, John Ole. 1990. Zur syntaktischen und referentiell-semantischen Typisierung der deutschen Pronominalform es. Deutsch als Fremdsprache 27. 213-225.

Behaghel, Otto. 1928. Deutsche Syntax. Eine geschichtliche Darstellung. Bd. 3. Die Satzgebilde. Heidelberg: Winter.

Bærentzen, Per. 1987. Die Spezialverwendungen des deutschen es und der dänischen Äquivalente det/der. Sprachwissenschaft 12. 341-380.

Corbett, Greville G. 1979. The agreement hierarchy. Linguistics 15. 203-224.

Eisenberg, Peter. 2013. Grundriss der deutschen Grammatik. Bd. 2. Der Satz. 4. Aufl. Stuttgart \& Weimar: Metzler.

Engel, Ulrich. 2009. Deutsche Grammatik. Neubearbeitung. 2. Aufl. München: Iudicium.

Helbig, Gerhard \& Joachim Buscha. 2017. Deutsche Grammatik. Ein Handbuch für den Ausländerunterricht. Stuttgart: Klett. Nachdruck der Ausgabe von 2001. Hentschel, Elke \& Harald Weydt. 2013. Handbuch der deutschen Grammatik. 4. Aufl. Berlin \& Boston: de Gruyter.

Krogh, Steffen. 2012. How Satmarish is Haredi Satmar Yiddish? In Marion Aptroot, Efrat Gal-Ed, Roland Gruschka \& Simon Neuberg (Hgg.), Yidishe shtudyes haynt. Jiddistik heute / Yiddish Studies Today, 483-506. Düsseldorf: Düsseldorf University Press.

Krogh, Steffen. 2015. Some remarks on the morphology and syntax of written Yiddish among Haredi Satmar Jews. In Michael Elmentaler, Markus Hundt \& Jürgen E. Schmidt (Hgg.), Deutsche Dialekte. Konzepte, Probleme, Handlungsfelder. Akten des 4. Kongresses der Internationalen Gesellschaft für Dialektologie des Deutschen (IGDD) in Kiel, 379-413. Stuttgart: Steiner.

Weinreich, Uriel. 1964. Western traits in Transcarpathian Yiddish. In For Max Weinreich on his seventieth birthday. Studies in Jewish languages, literature, and society, 245-264. London, The Hague \& Paris: Mouton \& Co.

Weinreich, Uriel. 1999. College Yiddish. An introduction to the Yiddish language and to Jewish life and culture. Sixth Revised Edition, First Printing. New York: YIVO.

Wöllstein, Angelika (Hg.). 2016. Duden. Die Grammatik. Unentbehrlich für richtiges Deutsch. 9. Aufl. Berlin: Dudenverlag.

Zifonun, Gisela, Ludger Hoffmann \& Bruno Strecker (Hgg.). 1997. Grammatik der deutschen Sprache. Bd. 1. Berlin \& New York: de Gruyter. 